\title{
Simulations of time-resolved $x$-ray diffraction in Laue geometry
}

\author{
B Lings ${ }^{1}$, J S Wark ${ }^{1,5}$, M F DeCamp ${ }^{2}$, D A Reis ${ }^{3}$ and S Fahy ${ }^{4}$ \\ ${ }^{1}$ Department of Physics, Clarendon Laboratory, University of Oxford, Oxford OX1 3PU, UK \\ 2 Department of Chemistry, Massachusetts Institute of Technology, Cambridge, MA 02139, USA \\ ${ }^{3}$ FOCUS Center and Department of Physics, University of Michigan, Ann Arbor, MI 48109, USA \\ ${ }^{4}$ Physics Department and NMRC, University College, Cork, Republic of Ireland
}

Received 12 March 2006, in final form 3 August 2006

Published 22 September 2006

Online at stacks.iop.org/JPhysCM/18/9231

\begin{abstract}
A method for computer simulation of time-resolved x-ray diffraction (TRXD) in asymmetric Laue (transmission) geometry with an arbitrary propagating strain perpendicular to the crystal surface is presented. We present two case studies for possible strain generation by short-pulse laser irradiation: (i) a thermoelastic-like analytical model; (ii) a numerical model including the effects of electron-hole diffusion, Auger recombination, deformation potential and thermal diffusion. A comparison with recent experimental results is also presented.
\end{abstract}

\section{Introduction}

When a semiconductor crystal is irradiated by photons of above band-gap energy, electrons are excited from the valence band to the conduction band. If the light source is a subpicosecond laser, the electron-hole plasma density can easily reach $10^{20} \mathrm{~cm}^{-3}$ - enough for the lattice spacing changes due to the deformation potential to be significant and for the fast diffusion of the electron-hole plasma into the crystal bulk to be appreciable. The timescale of the fastest energy transfer processes from the electrons to the lattice is of the order of picoseconds or less, which is significantly shorter than the hydrodynamic response time of the crystal. The resultant stress is therefore relieved by surface expansion and, by Newton's third law, a bipolar compression wave propagating into the crystal. These effects have been studied by optical methods [1-4] and, more recently, as test-cases for the field of time-resolved x-ray diffraction (TRXD) in Bragg (reflection) geometry [5-11]. However, although x-ray diffraction in Bragg geometry allows the direct study of the structural changes to the lattice-as opposed to the electronic effects in optical studies - the very nature of Bragg geometry x-ray diffraction with perfect crystals only allows the study of the surface region of the crystal. By utilizing the anomalous absorption effect in Laue (transmission) geometry, recent experiments [12, 13] have

5 http://laserplasma.physics.ox.ac.uk/ 
enabled the probing of structural changes throughout the bulk of the crystal on a subnanosecond timescale, giving a much clearer picture of the phenomena under consideration.

In Bragg geometry it is only possible to study the structural changes of crystalline matter in a layer with thickness comparable to the shorter of the x-ray extinction depth $(\sim \mu \mathrm{m})$ for strong diffraction or the absorption depth for weak diffraction [11]. In Laue geometry it is possible to study the whole bulk of the crystal due to anomalous absorption, known as the Borrmann effect. In dynamical diffraction theory, the x-ray electromagnetic field inside the crystal is resolved into two independent eigensolutions - one with nodes on the lattice planes, and the other with anti-nodes. This creates one solution with reduced absorption and another with enhanced absorption. These are known respectively as the $\alpha$ and $\beta$ branches. It is the $\alpha$ branch solution that can propagate for many extinction depths without significant lossprobing the entire depth of the crystal [14]. A disturbance in the crystal lattice causes energy to be transferred between the two branches, an effect known as inter-branch scattering [15]. When the x-ray beam exits the crystal, it is again expressed as the free-space solutions: the 0 (forward-diffracted) and $h$ (deflected-diffracted) beams, which are linear combinations of the $\alpha$ and $\beta$ branches. If the ratio and/or relative phases of the branches has changed, the energy partitioning between the beams will be affected, often to a significant degree [13].

Using this method it is possible to study the mechanism of energy transfer when a single crystal target is irradiated by a femtosecond laser pulse. We will show that it is possible to distinguish between a model which assumes an instantaneous energy transfer into the lattice and one which models thermal diffusion, electron-hole plasma diffusion, the effect of the deformation potential, and energy transfer to the lattice via Auger recombination.

\section{Theory}

The fundamental assumption in this method of diffraction simulation is that the strain field in the crystal is one-dimensional, parallel to the surface normal, and can be approximated by a constant strain in many laminae, each one parallel to the surface of the crystal. The degree to which this represents the experimental situation is discussed in section 3 . The electric displacement field amplitude is then calculated as it passes through these laminae, taking into account the effect of the strain in each.

\subsection{Dynamical diffraction theory}

By solving the wave equation

$$
\text { curl curl }[(1-\chi) \mathbf{D}(\mathbf{r})]=4 \pi^{2} k^{2} \mathbf{D}(\mathbf{r})
$$

in a medium with a periodic susceptibility

$$
\chi(\mathbf{r})=\sum_{h} \chi_{h} \mathrm{e}^{-2 \pi \mathrm{ih} \cdot \mathbf{r}}
$$

using a displacement field

$$
\mathbf{D}(\mathbf{r})=\sum_{h} \mathbf{D}_{h} \mathrm{e}^{-2 \pi \mathbf{i k}_{h} \cdot \mathbf{r}},
$$

where $\mathbf{k}_{h}=\mathbf{k}_{0}+\mathbf{h}$ is the wavevector of the $h$ th component inside the crystal and both are summed over Fourier components in the reciprocal lattice vectors $\mathbf{h}$, we obtain the fundamental equations of dynamical theory [16]

$$
\sum_{h^{\prime}} \chi_{h-h^{\prime}}\left[\left(\mathbf{k}_{h} \cdot \mathbf{D}_{h^{\prime}}\right) \mathbf{k}_{h}-k_{h}^{2} \mathbf{D}_{h^{\prime}}\right]=\left(k^{2}-k_{h}^{2}\right) \mathbf{D}_{h}
$$


These can be further simplified by the assumption that there are only two strong x-ray wavefields (incident and diffracted: $\mathbf{D}(\mathbf{r})=\mathbf{D}_{0} \mathrm{e}^{-2 \pi \mathbf{k}_{0} \cdot \mathbf{r}}+\mathbf{D}_{h} \mathrm{e}^{-2 \pi \mathrm{i} \mathbf{k}_{h} \cdot \mathbf{r}}$ ), leading to the two-beam dispersion relation

$$
\begin{aligned}
& \chi_{\bar{h}}\left[\left(\mathbf{k}_{0} \cdot \mathbf{D}_{h}\right) \mathbf{k}_{0}-k_{0}^{2} \mathbf{D}_{h}\right]=\left[k^{2}-k_{0}^{2}\left(1-\chi_{0}\right)\right] \mathbf{D}_{0}, \\
& \chi_{h}\left[\left(\mathbf{k}_{h} \cdot \mathbf{D}_{0}\right) \mathbf{k}_{h}-k_{h}^{2} \mathbf{D}_{0}\right]=\left[k^{2}-k_{h}^{2}\left(1-\chi_{0}\right)\right] \mathbf{D}_{h},
\end{aligned}
$$

where $\bar{h}$ indicates the direction inverse to $h$. These vector equations relate the physically realizable values of $\mathbf{D}_{0, h}$ and can be solved for the incident and diffracted beam amplitude as a function of depth into a crystal layer giving equations of the form (for $\sigma$-polarization)

$$
\left(\begin{array}{c}
D_{0} \\
D_{h}
\end{array}\right)=\left(\begin{array}{cc}
\mathrm{e}^{-2 \pi \mathbf{k}_{\alpha} \cdot \mathbf{r}} & \mathrm{e}^{-2 \pi \mathbf{i}_{\beta} \cdot \mathbf{r}} \\
\xi_{\alpha} \mathrm{e}^{-2 \pi i \mathbf{k}_{\alpha} \cdot \mathbf{r}} & \xi_{\beta} \mathrm{e}^{-2 \pi \mathbf{i}_{\beta} \cdot \mathbf{r}}
\end{array}\right)\left(\begin{array}{c}
D_{\alpha} \\
D_{\beta}
\end{array}\right),
$$

where

$$
\mathbf{k}_{\alpha} \stackrel{\text { def }}{=} \frac{k \delta_{0}^{\prime} \hat{\mathbf{n}}}{\gamma_{0}}, \quad \mathbf{k}_{\beta} \stackrel{\text { def }}{=} \frac{k \delta_{0}^{\prime \prime} \hat{\mathbf{n}}}{\gamma_{0}},
$$

are the wavevector shifts of the $\alpha$ and $\beta$ branches, $D_{\alpha}$ and $D_{\beta}$ are the amplitudes of the $\alpha$ and $\beta$ branches,

$$
\begin{aligned}
& \left.\begin{array}{l}
\delta_{0}^{\prime} \\
\delta_{0}^{\prime \prime}
\end{array}\right\}=\frac{1}{2}\left[\chi_{0}-z \pm \sqrt{q+z^{2}}\right] \\
& \left.\begin{array}{l}
\xi_{\alpha} \\
\xi_{\beta}
\end{array}\right\}=\frac{-z \pm \sqrt{q+z^{2}}}{\chi_{\bar{h}}}, \\
& z \stackrel{\text { def }}{=} \frac{1-b}{2} \chi_{0}-b \Delta \theta \sin 2 \theta_{\mathrm{B}}, \quad q \stackrel{\text { def }}{=} b \chi_{h} \chi_{\bar{h}}, \\
& b \approx \frac{\gamma_{0}}{\gamma_{h}}, \quad \Delta \theta=\theta-\theta_{\mathrm{B}},
\end{aligned}
$$

where $\gamma_{0}, \gamma_{h}$ are the direction cosines of the incoming and outgoing beams, respectively, in relation to the surface normal of the crystal $\hat{\mathbf{n}} ; \xi_{\alpha, \beta}$ are the amplitude ratios $D_{h} / D_{0} ; \theta$ is the actual glancing angle of incidence relative to the diffracting planes; and $\theta_{\mathrm{B}}$ is the Bragg glancing angle [16]. One can also define a dimensionless deviation parameter $\eta$, defined as

$$
\eta=\frac{\Delta \theta-\Delta \theta_{o s}}{\delta_{o s}}
$$

where $\Delta \theta_{o s}$ is the refraction shift of the diffraction peak and $2 \delta_{o s}$ is the Darwin width [15]. This can be shown to be equal to $-\frac{z}{\sqrt{q}}$ in terms of the quantities shown above. The deviation parameter can also be expressed as a function of wavelength separation from the Bragg wavelegth $\Delta \lambda=\lambda-\lambda_{\mathrm{B}}$, using the relation $\Delta \lambda=\left(\lambda_{\mathrm{B}} \cot \theta_{\mathrm{B}}\right) \Delta \theta$ derived from Bragg's law. This is the form that will be used for analysing the experimental data. However, it is easier to visualize the effects of strain (see the following section) when it is considered as a function of angle.

Equation (4) can be solved at the boundary between two layers $(\hat{\mathbf{n}} \cdot \mathbf{r}=0)$ to find $D_{\alpha}$ and $D_{\beta}$ as functions of $D_{0}$ and $D_{h}$ in the previous layer:

$$
\left(\begin{array}{c}
D_{\alpha} \\
D_{\beta}
\end{array}\right)^{(i+1)}=\frac{1}{\xi_{\beta}-\xi_{\alpha}}\left(\begin{array}{cc}
\xi_{\beta} & -1 \\
-\xi_{\alpha} & 1
\end{array}\right)\left(\begin{array}{c}
D_{0} \\
D_{h}
\end{array}\right)^{(i)}
$$

where the superscripts indicate the layer in question. These layers are illustrated in figure 1. In order to propagate the x-rays through the crystal, equation (11) is evaluated at the layer boundaries, and the $D_{0}$ and $D_{h}$ amplitudes at the end of the layer are calculated using 


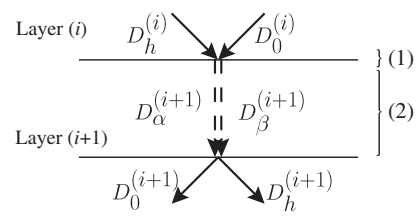

Figure 1. Layer approximation. (1) At the boundaries between layers, the $\alpha$ and $\beta$ branches are calculated (equation (11)). (2) The beam is then propagated through the layer (equation (4)).

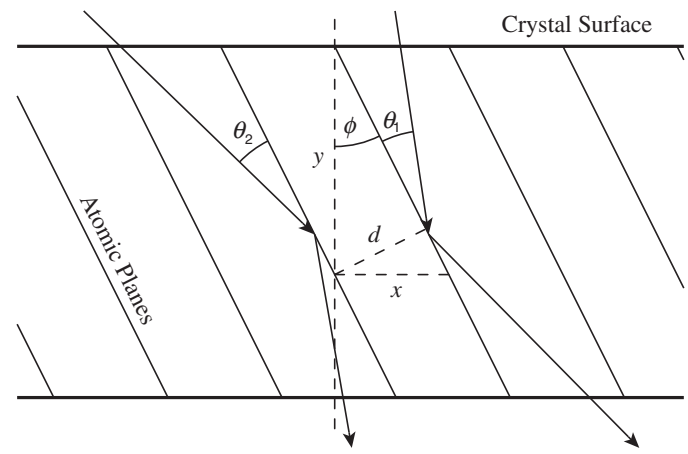

Figure 2. Strained crystals. Strain $\left(\epsilon_{\perp}=\frac{\delta y}{y}\right)$ alters the rotation $(\delta \phi)$ and the separation $(\delta d)$ of lattice planes in the crystal. Note that there are two possible orientations for the $\mathrm{x}$-ray beams.

equation (4). It should be noted that $\mathbf{k}_{\alpha, \beta}$ and $\xi_{\alpha, \beta}$ are all functions of $\eta$, and it is through this parameter that the strain is incorporated. Therefore, in equation (11), care must be taken that the $\xi_{\alpha, \beta}$ are evaluated in the correct layer. We may do so by considering each layer as a separate crystal, with the beams $D_{0, h}^{(i)}$ exiting crystal $(i)$ and then entering crystal $(i+1)$ without change. It is then easy to see that the correct layer in which to evaluate these quantities is layer $(i+1)$.

\subsection{Incorporating strain}

Strain is incorporated into the model through the deviation parameter $\eta$ (or more specifically, its real part $\eta_{\mathrm{r}}$ ), which can be expressed as a function of the angular deviation from the Bragg angle $\Delta \theta$ (equation (10)). Strain affects this angular deviation in two ways: (i) rotating the planes (changing $\theta$ ) and (ii) altering the separation (changing $\theta_{\mathrm{B}}$ ). The method presented here is similar to that of earlier work in Bragg geometry [17-19], but is now presented for the Laue geometry case.

From figure 2 we can formulate the following relations by taking differentials of the relationships between $d, x, y$ and $\phi$ :

$$
\begin{aligned}
& \delta \phi=-\epsilon_{\perp} \sin \phi \cos \phi, \\
& \frac{\delta d}{d}=\epsilon_{\perp} \sin ^{2} \phi,
\end{aligned}
$$

where $\epsilon_{\perp}=\frac{\delta y}{y}$ is the strain perpendicular to the surface.

The change in diffraction angle $\delta \theta$ is equal to $\pm \delta \phi$ depending on the orientation of the incoming and outgoing beams ( $\theta_{1}$ or $\theta_{2}$ in figure 2 for positive and negative $\phi$, respectively). The change in diffraction angle due to the planar rotation is therefore

$$
\delta \theta=\mp \epsilon_{\perp} \sin \phi \cos \phi .
$$


The change in the Bragg angle can be calculated using the differential form of Bragg's law,

$$
\delta \theta_{\mathrm{B}}=\frac{\delta d}{d} \tan \theta_{\mathrm{B}}
$$

combined with equation (13), giving

$$
-\delta \theta_{\mathrm{B}}=\epsilon_{\perp} \sin ^{2} \phi \tan \theta_{\mathrm{B}}
$$

We can now include both of these effects in the angular deviation from the Bragg angle to give the 'effective misorientation'

$$
\left(\theta-\theta_{\mathrm{B}}\right) \rightarrow\left(\theta-\theta_{\mathrm{B}}\right)+\left(\sin ^{2} \phi \tan \theta_{\mathrm{B}} \mp \sin \phi \cos \phi\right) \epsilon_{\perp} .
$$

This can be used in every lamina, with the appropriate strain, in order to calculate the timeresolved rocking curves from the strained crystal. In order for this lamellar approximation to be considered accurate, each lamina must be sufficiently thin that the change in the effective misorientation is small within it. In practice this can be achieved by using a variable layer thickness, with a maximum allowed strain change across a layer. The maximum strain change within a layer can then be reduced until the results obtained remain constant. This was achieved with a maximum strain change of $1 \times 10^{-8}$ within any layer, for the geometry under consideration in this paper.

\section{Strain models}

As an example of this technique, we use the case of a germanium single crystal irradiated by a femtosecond pulse of near infra-red laser radiation. Experiments using Laue geometry timeresolved $x$-ray diffraction on such a sample have recently been carried out [12]. This case has also been studied experimentally using ultrafast reflectivity [1-4] and x-ray Bragg scattering techniques [5-11].

In this experimental set-up, the short laser pulse quickly heats the surface at constant volume, generating a thermal stress. This causes the surface to expand and, by Newton's third law, launches an acoustic pulse into the crystal. In both cases considered here, the lateral size of the laser spot is assumed to be much greater than the laser absorption depth. As a result, the strain generated can be assumed to be one dimensional ${ }^{6}$, i.e. the atomic displacement only varying as a function of depth into the crystal.

We will be comparing two models of the strain produced in this system. The first model takes into account the timescales of the processes by which the energy is transferred to the lattice. The second is a simplified case with an analytical solution, introduced by Thomsen et al [20]. It assumes instantaneous transfer of energy from the laser into the lattice and no diffusion.

When a short laser pulse is incident on the crystal, the following energy transfer processes occur (see figure 3):

(1) The laser excites electrons from the valence band to the conduction band of the semiconductor, creating an electron-hole plasma.

(2) The electrons and holes quickly relax to the band edges, transferring their energy to the lattice. This energy transfer is assumed to be instantaneous on the timescale considered $(\lesssim 1 \mathrm{ps})$.

6 This is for times less than lateral extent of the spot divided by the speed of sound. This corresponds to hundreds of nanoseconds for the experimental conditions modelled. 


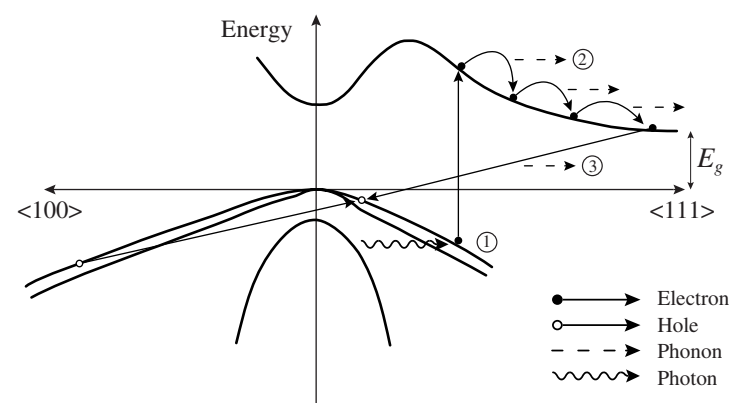

Figure 3. Band structure of germanium. The transfer of energy to the lattice involves: (1) laser excitation, (2) fast relaxation to band edges, (3) Auger recombination.

(3) The electrons and holes then recombine (by Auger recombination-an e-e- $\mathrm{h}$ and $\mathrm{e}-\mathrm{h}-\mathrm{h}$ process), transferring the rest of the energy to the lattice [21]. This takes significantly longer (typically $1 \mathrm{~ns}$ to $1 \mu \mathrm{s}$ ), allowing the carriers to diffuse appreciably into the crystal before recombining.

The electron-hole plasma and the thermal phonons in the lattice both diffuse into the crystal bulk, with separate diffusion constants. They both cause a change in the lattice spacing, through the deformation potential and thermal expansion, respectively.

The laser energy is deposited in a certain area of the crystal with a 1/e absorption depth $\zeta$ and an absorbed fluence $Q$. This gives initial conditions of the electron-hole plasma and the lattice temperature as

$$
\begin{aligned}
& n(z, t=0)=\frac{Q}{E_{\mathrm{p}} \zeta} \mathrm{e}^{-z / \zeta}, \\
& T(z, t=0)=\frac{E_{\mathrm{p}}-E_{\mathrm{g}}}{C_{\mathrm{l}}} n(z, t=0),
\end{aligned}
$$

where $n$ is the electron-hole plasma density, $E_{\mathrm{p}}$ is the energy of the laser photons, $E_{\mathrm{g}}$ is the indirect band gap and $C_{1}$ is the lattice heat capacity per unit volume. The electron-hole plasma obeys a diffusion equation with a sink term for Auger recombination

$$
\frac{\partial n}{\partial t}=D_{\mathrm{p}} \frac{\partial^{2} n}{\partial z^{2}}-A n^{3}
$$

where $D_{\mathrm{p}}$ is the plasma diffusion constant and $A$ is the Auger recombination rate. The energy from the Auger recombination is then transferred to the lattice, which obeys a diffusion equation with a corresponding source term

$$
\frac{\partial T}{\partial t}=D_{\mathrm{t}} \frac{\partial^{2} T}{\partial z^{2}}+A n^{3} \frac{E_{\mathrm{g}}}{C_{\mathrm{l}}},
$$

where $D_{\mathrm{t}}$ is the thermal diffusion constant.

The equilibrium strain is then

$$
\epsilon_{\mathrm{e}}(z, t)=\alpha_{\mathrm{t}} T(z, t)+\alpha_{\mathrm{p}} n(z, t),
$$

where $\alpha_{\mathrm{t}}$ is the thermal expansivity $(=\Phi \beta, \Phi$ is a factor to take into account the 1D nature of the strain, $\beta$ is the linear expansion coefficient) and $\alpha_{\mathrm{p}}$ is an electronic contribution to the strain associated with the deformation potential $(=\partial(\log a) / \partial n, a$ is the equilibrium lattice 


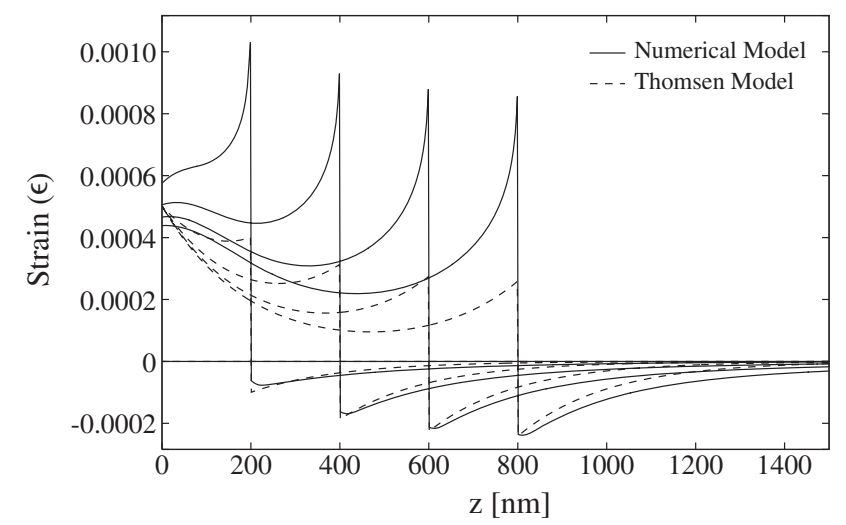

Figure 4. Strain profile evolution-comparison of numerical and Thomsen models. This figure shows a time sequence of strain snapshots taken at $\sim 40 \mathrm{ps}$ intervals $\left(v=4915 \mathrm{~m} \mathrm{~s}^{-1}\right)$ for an absorbed fluence of $Q=4 \mathrm{~mJ} \mathrm{~cm}^{-2}$. The physical parameters used are the literature values given in section 6. The strain in the numerical model comprises a diffusing surface component and an asymmetric travelling wave. The asymmetry is caused by the $\mathrm{e}-\mathrm{h}$ diffusion, the higher peak strain by the deformation potential, and the surface decay by a combination of thermal and $\mathrm{e}-\mathrm{h}$ diffusion. The strain in the Thomsen model is made up of an exponential surface component and an antisymmetric travelling wave. The travelling portions of the strain move into the crystal at the speed of sound.

constant). Any change in the equilibrium strain produces forward and backward propagating waves, which can be calculated by integrating equation (21) as follows:

$$
\begin{aligned}
& \epsilon_{+}(z, t)=-\frac{1}{2} \int_{0}^{t} \frac{\partial}{\partial t}\left[\epsilon_{\mathrm{e}}\left(z-v t^{\prime}, t-t^{\prime}\right)\right] \mathrm{d} t^{\prime}, \\
& \epsilon_{-}(z, t)=-\frac{1}{2} \int_{0}^{t} \frac{\partial}{\partial t}\left[\epsilon_{\mathrm{e}}\left(z+v t^{\prime}, t-t^{\prime}\right)\right] \mathrm{d} t^{\prime},
\end{aligned}
$$

where $v$ is speed of longitudinal sound in the crystal. The free-surface boundary condition of the stress, $\sigma_{33}(z=0, t) \equiv 0$, is ensured by defining

$$
\epsilon_{\mathrm{e}}(-z, t) \equiv-\epsilon_{\mathrm{e}}(z, t), \quad z>0 .
$$

The total strain is then the sum of the equilibrium and the forward and backward going strain waves.

$$
\epsilon(z, t)=\epsilon_{\mathrm{e}}(z, t)+\epsilon_{+}(z, t)+\epsilon_{-}(z, t) .
$$

The Thomsen strain model [20] in its most simple form assumes instantaneous transfer of energy into the lattice and no diffusion. In the timescale of interest only a fraction $\left(E_{\mathrm{p}}-E_{\mathrm{g}}\right) / E_{\mathrm{p}}$ of the absorbed laser energy is transferred to the lattice (the rest remaining in the electron-hole plasma). This gives a total strain of

$\epsilon_{\mathrm{Th}}(z, t)=\frac{Q \Phi \beta\left(E_{\mathrm{p}}-E_{\mathrm{g}}\right)}{\zeta C_{\mathrm{l}} E_{\mathrm{p}}}\left\{\mathrm{e}^{-z / \zeta}-\frac{1}{2}\left[\mathrm{e}^{-(z+v t) / \zeta}+\mathrm{e}^{-|z-v t| / \zeta} \operatorname{sgn}(z-v t)\right]\right\}$.

Note that this is the sum of a non-evolving exponential surface strain, a forward going wave $\mathcal{F}(z-v t)$ and a backward going wave $\mathcal{G}(z+v t)$.

The numerical model produces an identical strain to the above analytical formula, if there is no deformation potential or Auger recombination $\left(\alpha_{\mathrm{p}}=0\right.$ and $\left.A=0\right)$. A comparison of the strains produced by the numerical and Thomsen strain models is shown in figure 4 . 



Figure 5. Simulated time-resolved rocking curves. The 0 and $h$ beam rocking curves plotted as a function of time for the numerical strain model with an absorbed pump fluence of $Q=17 \mathrm{~mJ} \mathrm{~cm}^{-2}$.

(This figure is in colour only in the electronic version)

\section{Simulation}

The diffraction simulation works as follows: Over a number of time-steps, the strain profile (analytical or numerical) is calculated. For the numerical method, the diffusion equations (19) and (20) are solved using a Crank-Nicholson scheme, and the integrals in equation (22) are calculated by finite differencing then summing. The x-rays are then propagated through the crystal over the $\eta_{\mathrm{r}}$ range of interest. This is done using the propagation matrices (section 2.1). After the final layer, the amplitudes for the 0 and $h$ beams are multiplied by their complex conjugates, giving the rocking curves (figure 5). The resulting time-resolved rocking curves are then integrated over $\eta_{\mathrm{r}}$ and normalized such that the value for an unstrained crystal is unity, for comparison with experimental results.

It is also possible to output the beam intensities through the bulk of the crystal: either as the 0 and $h$ beams, showing Pendellösung oscillations [14], or as the $\alpha$ and $\beta$ branches, showing the transfer of energy between the two as the strain moves through the crystal (see figure 9).

\section{Experiment}

The experimental data were taken at the 7-ID Undulator Beamline at the Advanced Photon Source. The x-ray beam energy was $10 \mathrm{keV}$ with a $1.4 \times 10^{-4}$ fractional energy spread (larger than the rocking curve width of the crystal) and negligible beam divergence. The sample was a $280 \mu \mathrm{m}$ thick (001) germanium single crystal, oriented to diffract from the $20 \overline{2}$ planes (figure 6). The x-ray beam was masked by tantalum slits giving a beam size on the crystal of $400 \mu \mathrm{m} \times 400 \mu \mathrm{m}$. The strain pulse was produced by exciting the output face of the crystal 




Figure 6. Experimental setup. The $h$ (deflected-defracted) beam is collected by an avalanche photodiode (APD) giving a time resolution of the synchrotron bunch length, the 0 (forwarddiffracted) beam is resolved by a streak camera giving picosecond resolution.

with sub-100 fs, $800 \mathrm{~nm}$ laser pulses. The diffraction simulation was set up to match these experimental conditions.

The time sequence of $\mathrm{x}$-ray measurements was built up using a pump-probe scheme. The relative timing of the $\mathrm{x}$-ray and laser pulses was achieved by a variable electronic delay, set with a precision of 19 ps. Thus, each time-step shown in the results is the delay between the laser and x-ray pulses reaching the crystal. Each x-ray pulse, of 100 ps duration, illuminates the crystal with an effectively static strain profile.

\section{Results and discussion}

Data taken experimentally [12] are shown in figure 7(a). Oscillations are seen in the $h$ beam amplitude, with the time evolution of these varying with pump fluence. The phase of the oscillations is calculated by fitting curves of the form

$$
I(t)=\sum_{i} a_{i} \mathrm{e}^{-\frac{t}{b_{i}}} \sin \left(\omega_{i} t-\phi_{i}\right),
$$

where $t$ is the time after laser excitation, starting at $t=1 \mathrm{~ns}$ (to ignore the initial rise). The size of the initial rise or fall is measured by taking the normalized intensity at $t=200 \mathrm{ps}$. The phase $\phi$ and the initial rise or fall $\left(\left|D_{h}(t=200 \mathrm{ps})\right|^{2} /\left|D_{h}(t=0 \mathrm{ps})\right|^{2}\right)$ of the measured and simulated reflectivity is shown in figure 8 . The curves for measured reflectivity assume that $75 \%$ of the laser energy is absorbed by the crystal.

Simulations were run with the Thomsen strain model, using literature values for physical parameters $\left(\zeta=200 \mathrm{~nm}, E_{\mathrm{g}}=0.67 \mathrm{eV}, \Phi \beta=1 \times 10^{-5} \mathrm{~K}^{-1}, C_{\mathrm{l}}=1.7 \mathrm{~J} \mathrm{~K}^{-1} \mathrm{~cm}^{-3}\right)$. The results are shown in figures 7 and $8(\mathrm{~b})$. These do not match well with either the phase of the oscillations, the initial rise or fall, or the overall form of the $h$ beam intensity. If the laser absorption depth $\zeta$ is increased to $1 \mu \mathrm{m}$ (figures 7 and 8(c)), the overall form of the higher fluence curves shows a better match. However, neither the phase of the oscillations nor the initial rise or fall show the expected behaviour.

Using the numerical model, but only adding the effects of thermal diffusion (no deformation potential or Auger recombination), as shown in figures 7 and 8(d), a better match for the phase of oscillations is achieved. This now agrees with the experimentally measured phase to approximately $10 \%$. The initial behaviour, however, remains badly matched.

Using the more detailed numerical model, with literature values for all physical parameters $\left(\zeta=200 \mathrm{~nm}, \alpha_{\mathrm{t}}=1 \times 10^{-5} \mathrm{~K}^{-1}, \alpha_{\mathrm{p}}=1.3 \times 10^{-24} \mathrm{~cm}^{3}, A=1.1 \times 10^{-31} \mathrm{~cm}^{6} \mathrm{~s}^{-1}\right.$, $\left.D_{\mathrm{t}}=0.35 \mathrm{~cm}^{2} \mathrm{~s}^{-1}, D_{\mathrm{p}}=65 \mathrm{~cm}^{2} \mathrm{~s}^{-1}\right)$, the curves in figures 7 and $8(\mathrm{e})$ were calculated. These match the fluence dependence of the experiment in the overall form of the curves and the initial rise. However, the phase of the oscillations only decreases at about two-thirds the rate of the 

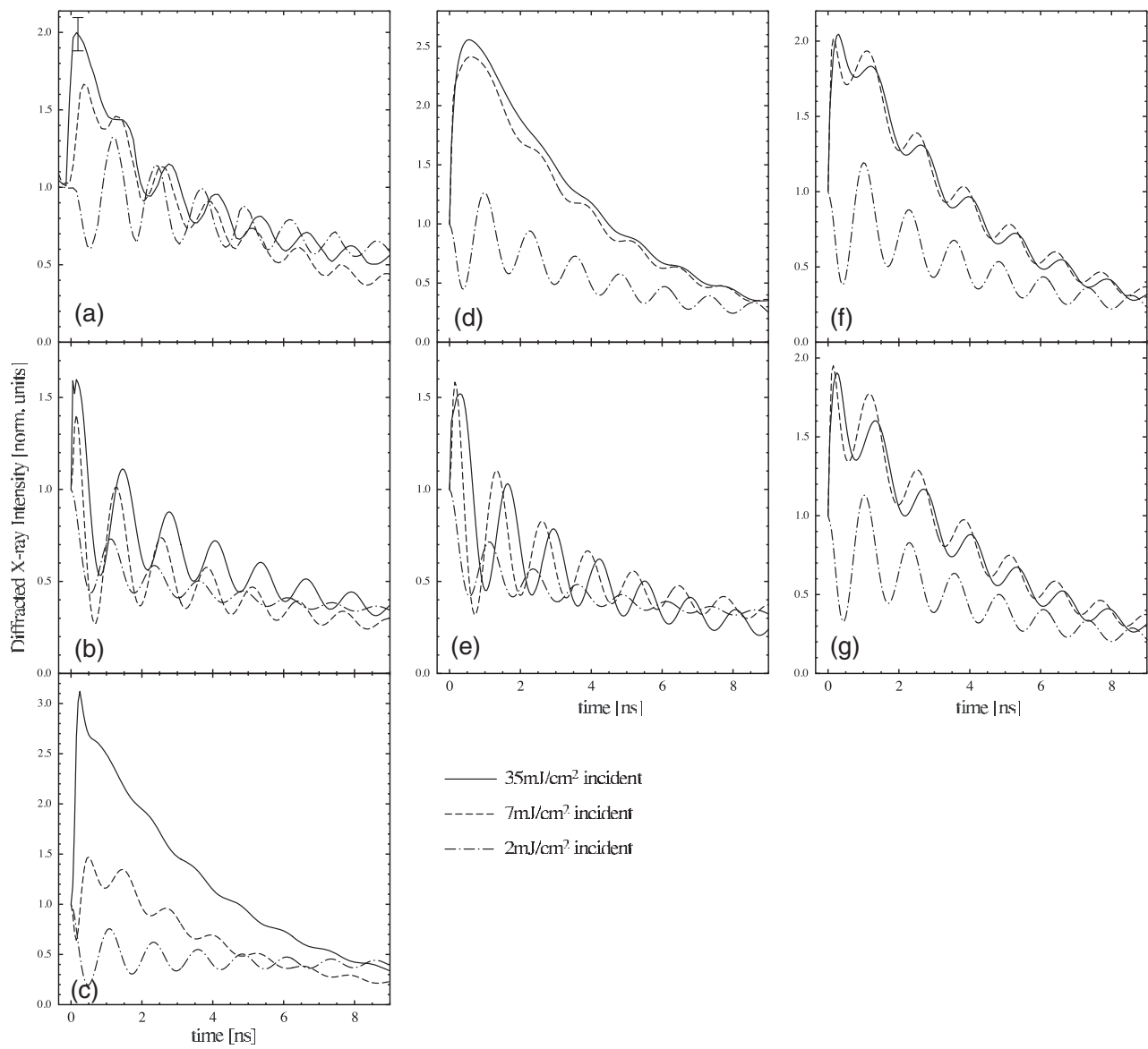

Figure 7. Time-resolved integrated reflectivity. Plots are for a diffracted beam $\left(\left|D_{h}\right|^{2}\right)$. (a) Experimental results. (b) Thomsen model with $\zeta=200 \mathrm{~nm}$. (c) Thomsen model with $\zeta=1 \mu \mathrm{m}$. (d) Numerical model, only diffusion included, $D_{\mathrm{t}}=0.35 \mathrm{~cm}^{2} \mathrm{~s}^{-1}, D_{\mathrm{p}}=0$, $A=0, \alpha_{\mathrm{t}}=10^{-5} \mathrm{~K}^{-1}, \alpha_{\mathrm{p}}=0$. (e) Numerical model, $D_{\mathrm{t}}=0.35 \mathrm{~cm}^{2} \mathrm{~s}^{-1}, D_{\mathrm{p}}=65 \mathrm{~cm}^{2} \mathrm{~s}^{-1}$, $A=1.1 \times 10^{-31} \mathrm{~cm}^{6} \mathrm{~s}^{-1}, \alpha_{\mathrm{t}}=10^{-5} \mathrm{~K}^{-1}, \alpha_{\mathrm{p}}=1.3 \times 10^{-24} \mathrm{~cm}^{3}, \zeta=200 \mathrm{~nm}, E_{\mathrm{g}}=0.67 \mathrm{eV}$, $C_{\mathrm{l}}=1.7 \mathrm{~J} \mathrm{~K}^{-1} \mathrm{~cm}^{-3}$ and $v=4915 \mathrm{~m} \mathrm{~s}^{-1}$. (f) Numerical model with no deformation potential, as (e) but with $\alpha_{\mathrm{p}}=0$. (g) and (h) Numerical model with increased Auger recombination, as (e) but with $A=5.5 \times 10^{-31} \mathrm{~cm}^{6} \mathrm{~s}^{-1}$ and $11 \times 10^{-31} \mathrm{~cm}^{6} \mathrm{~s}^{-1}$ respectively. Note that the modelling assumes that $75 \%$ of the incident laser energy is absorbed.

experimentally measured phase. At the highest fluences the oscillations in $D_{h}$ are of such small magnitude that it was impossible to fit the curves numerically.

If the deformation potential is taken out of the numerical model (by setting $\alpha_{\mathrm{p}}=0$ ), the correct fluence dependence of the phase is obtained (to approximately 10\%), but the behaviour at early times (i.e. the initial rise or fall) is no longer well matched (figures 7 and 8(f)).

These last two observations would imply that there is a direct electronic contribution to the strain (through the deformation potential) at earlier times, which is greatly reduced at later times. One possible mechanism for this is that the Auger recombination rate is larger than expected. Simulations were run with the recombination rate increased five-fold and ten-fold ( $A=5.5 \times 10^{-31} \mathrm{~cm}^{6} \mathrm{~s}^{-1}$ and $11 \times 10^{-31} \mathrm{~cm}^{6} \mathrm{~s}^{-1}$, respectively). The resulting curves (figures 7, 8(g) and (h), respectively) show good agreement with the experimental phase, and a reasonable agreement with early time behaviour. 


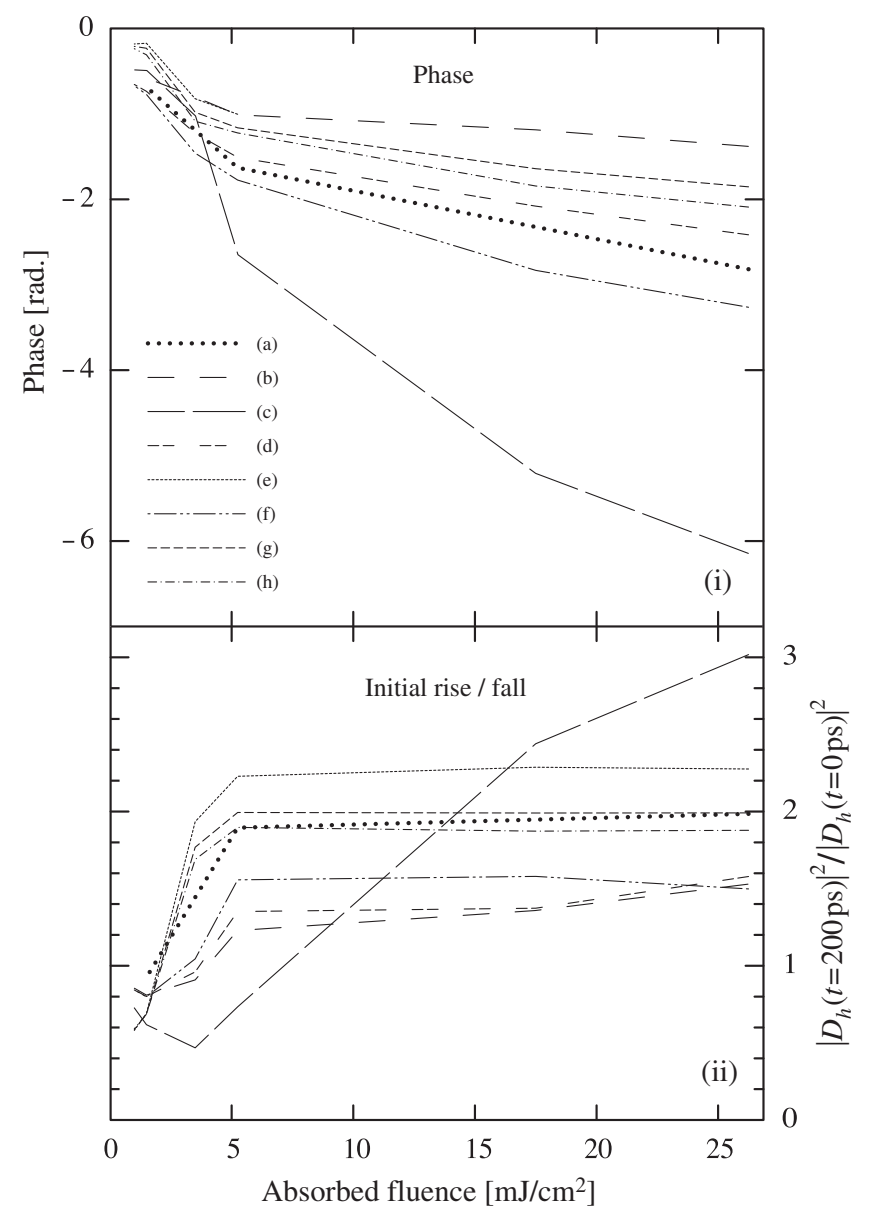

Figure 8. Effects of changing the deformation potential and the plasma diffusion constant on (i) the phase of the oscillations and (ii) the initial rise or fall of $\left|D_{h}\right|^{2}$. The phase is fitted to curves of the form shown in equation (25), with the data starting at $t=1 \mathrm{~ns}$ to ignore the initial rise. Fitted to: (a) Experimental data (assuming 75\% absorption). (b) Thomsen strain model, $\zeta=200 \mathrm{~nm}$. (c) Thomsen model, $\zeta=1 \mu \mathrm{m}$. (d) Numerical strain model, with only thermal diffusion included, $\alpha_{\mathrm{t}}=1 \times 10^{-5} \mathrm{~K}^{-1}, \alpha_{\mathrm{p}}=0, A=0$. (e) Numerical strain model, $\alpha_{\mathrm{t}}=1 \times 10^{-5} \mathrm{~K}^{-1}$, $\alpha_{\mathrm{p}}=1.3 \times 10^{-24} \mathrm{~cm}^{3}, A=1.1 \times 10^{-31} \mathrm{~cm}^{6} \mathrm{~s}^{-1}$. (f) Numerical model with no deformation potential, as (e) but with $\alpha_{\mathrm{p}}=0$. (g) and (h) Numerical model with increased Auger recombination, as (e) but with $A=5.5 \times 10^{-31} \mathrm{~cm}^{6} \mathrm{~s}^{-1}$ and $11 \times 10^{-31} \mathrm{~cm}^{6} \mathrm{~s}^{-1}$, respectively.

We were unable to find an excellent match with any combination of parameters, but this particular set gave the best of those tried. The exact mechanism for a larger than expected Auger recombination rate is unknown. One possible explanation is that the higher temperature of the lattice at early times activates other possible recombination pathways (leading to a temperature dependence to the recombination rate). It is thought that the main reason for the recombination rate in germanium being so low is that it requires phonon activation [22]. So, at the higher temperatures existing at early times, this could cause faster than expected recombination. More detailed modelling of the strain, taking this possible non-linearity into account, could lead to better reproduction of the experimental data.

In an earlier paper [12], the authors posited that the basic physics behind the oscillations visible in the $\eta$-integrated rocking curves (figure 7) was due to energy being transferred from the 


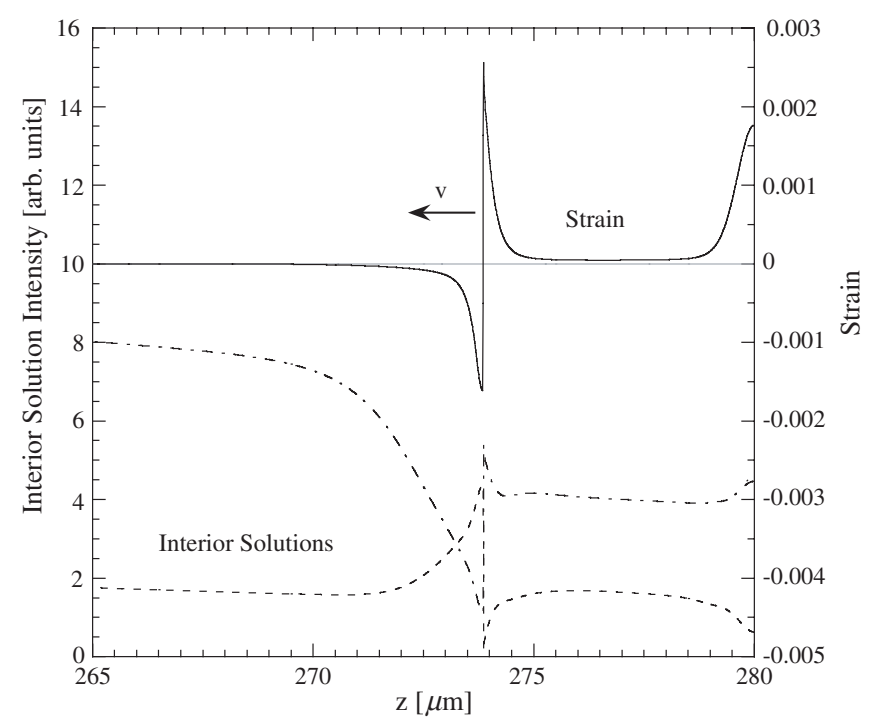

Figure 9. The $\eta$-integrated interior solutions $\left|D_{\alpha}\right|^{2}$ (dot-dashed line) and $\left|D_{\beta}\right|^{2}$ (dashed line) as a function of depth into the crystal at $1.25 \mathrm{~ns}$ after laser excitation, for an absorbed pump fluence of $Q=17 \mathrm{~mJ} \mathrm{~cm}^{-2}$. Also shown is the strain distribution (solid line). The strain front is moving from right to left. X-ray beams exit crystal on right.

$\alpha$ branch to the strongly absorbed $\beta$ branch at a disturbance in the lattice. Using the diffraction simulation it is possible to look at how the strain transfers this energy between the solutions. Figure 9 shows that energy is transferred from the $\alpha$ branch to the $\beta$ branch when the strain gradient is negative, and is then quickly transferred back at the positive strain discontinuity. This energy transfer effect is reversed for the opposite asymmetry $(\overline{2} 02)$.

It might initially be expected that analysis of these experimental results would need a $2 \mathrm{D}$ diffraction model. This is because, as shown in figure 10, for this experiment only a small fraction of the exit surface is influenced by the entire incoming x-ray beam-the area of influence being the inverted Borrmann triangle from the exit point [15]. The 2D Takagi-Taupin equations normally reduce to a case dependent only on the depth into the crystal for a 1D strain, as in this case [23]. However, this also has the implicit assumption that the lateral extent of the $\mathrm{x}$-ray beam is large enough for its size to be unimportant. Two-dimensional simulations were run, using the 'half-step derivative' numerical solution [24]. Sample results are shown in figure 11. For comparison with the 1D results, the intensity distribution was summed over the exit surface of the crystal, and then over the rocking curve. The time-resolved integrated reflectivities obtained only differed by $0.25 \%$ (RMS) from the 1D diffraction model. The reason for this is that close to the rocking curve peak, the $D_{0, h}$ beams are not 'eigensolutions' inside the crystal- the $D_{\alpha, \beta}$ solutions are, due to the strong coupling between the beams. We would therefore only expect differences to become apparent in the rocking curve wings, where the coupling is less strong. In the experimental conditions, where the data obtained are the $\eta$ integrated rocking curve, the great majority of the signal comes from the rocking curve peak, completely swamping any small changes in the wings.

\section{Conclusion}

We have presented a method for the calculation of time-resolved rocking curves for x-ray diffraction in Laue (transmission) geometry. Such diffraction studies are an important technique 


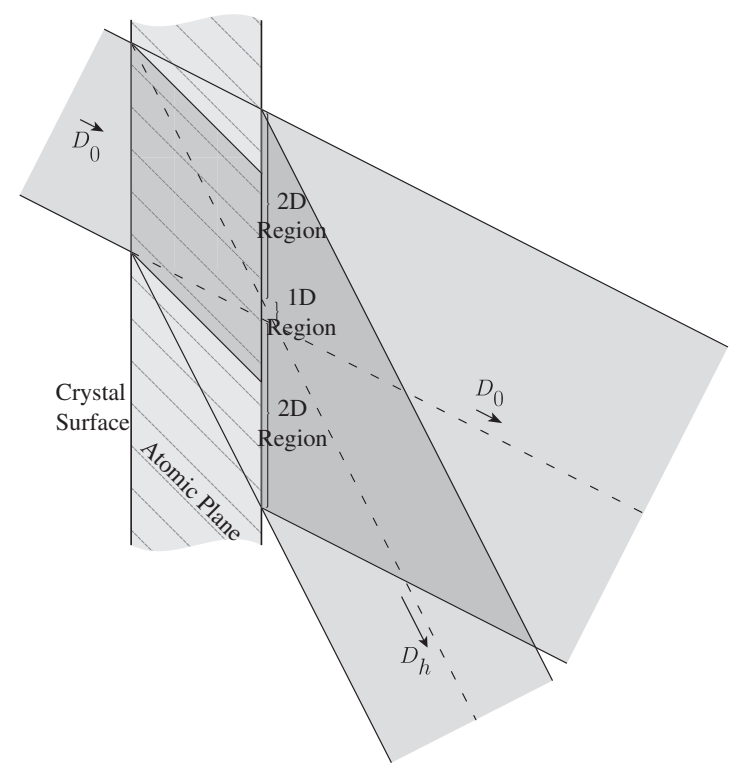

Figure 10. Two-dimensional region (drawn to scale). The incoming $D_{0}$ beam has a width of $400 \mu \mathrm{m}$, the crystal is $280 \mu \mathrm{m}$ thick. In the '2D region' shown, the exit surface of the crystal is not influenced by the whole incoming beam.

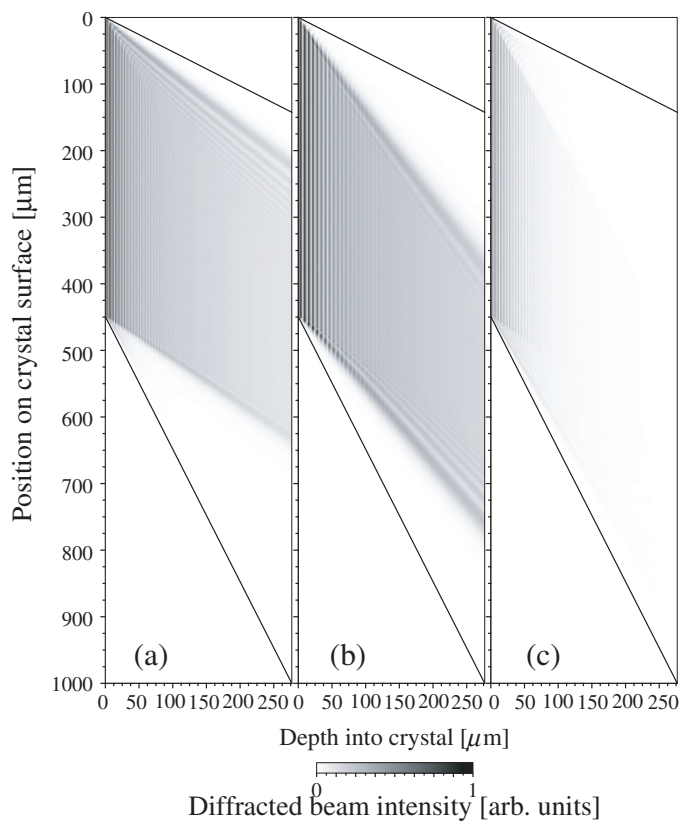

Figure 11. Two-dimensional simulation results (drawn to scale). $\left|D_{h}\right|^{2}$ within the crystal for (a) $\eta_{\mathrm{r}}=-1$, (b) $\eta_{\mathrm{r}}=0$, (c) $\eta_{\mathrm{r}}=+1$.

for the study of coherent strain in crystals beyond the extinction depth. Even without the benefit of rocking curve resolution of the diffracted x-ray beams, it is possible to obtain valuable 
information about the form of the strain profile in the crystal, and to observe the effects of the mechanisms of the ultrafast energy transfer processes at work. As there is no method to analytically compute the strain in a crystal from a time-resolved rocking curve, numerical simulations are key to the understanding of experimental results obtained.

\section{Acknowledgments}

The experimental work was conducted at the MHATT-CAT insertion device beam-line at the Advanced Photon Source and was supported in part by the US Department of Energy, grant nos DE-FG02-99ER45743 and DE-FG02-00ER15031 and from the NSF FOCUS Physics Frontier Center. Use of the Advanced Photon Source was supported by the US Department of Energy Basic Energy Sciences, Office of Energy Research under contract no W-31-109-Eng38. SF acknowledges the financial support of Science Foundation Ireland. BL acknowledges the financial support of EPSRC and AWE.

\section{References}

[1] Chigarev N V, Paraschuk D Yu, Pan X Y and Gusev V E 2000 Phys. Rev. B 6115837

[2] Auston D H and Shank C V 1974 Phys. Rev. Lett. 321120

[3] Auston D H, Shank C V and LeFur P 1975 Phys. Rev. Lett. 351022

[4] Tanaka K, Ohtake H, Nansei H and Suemoto T 1995 Phys. Rev. B 5210709

[5] Rose-Petruck C, Jimenez R, Guo T, Cavalleri A, Siders C W, Ráski F, Squier J A, Walker B C, Wilson K R and Barty C P J 1999 Nature 398310

[6] Cavalleri A et al 2001 Phys. Rev. B 63193306

[7] Siders C W, Cavalleri A, Sokolowski-Tinten K, Tóth Cs, Guo T, Kammler M, Horn von Hoegen M, Wilson K R, von der Linde D and Barty C P 1999 Science 2861340

[8] Cavalleri A et al 2000 Phys. Rev. Lett. 85586

[9] Sokolowski-Tinten K, Blome C, Dietrich C, Tarasevitch A, Horn von Hoegen M, von der Linde D, Cavalleri A, Squier J and Kammler M 2001 Phys. Rev. Lett. 87225701

[10] Lindenberg A M et al 2000 Phys. Rev. Lett. 84111

[11] Reis D A et al 2001 Phys. Rev. Lett. 863072

[12] DeCamp M F et al 2003 Phys. Rev. Lett. 91165502

[13] DeCamp M F et al 2001 Nature 413825

[14] Batterman B W and Cole H 1964 Rev. Mod. Phys. 36681

[15] Authier A 2001 Dynamical Theory of X-Ray Diffraction (Oxford: Oxford University Press) ISBN 0-19-855960-7

[16] Zachariasen W H 1945 Theory of X-Ray Diffraction in Crystals (New York: Dover) ISBN 0-486-68363-X

[17] Speriosu V S 1981 J. Appl. Phys. 526094

[18] Speriosu V S and Vreeland T Jr 1984 J. Appl. Phys. 561591

[19] Wie C R, Tombrello T A and Vreeland T 1986 J. Appl. Phys. 593743 (see erratum [25])

[20] Thomsen C, Grahn H T, Maris H J and Tauc J 1986 Phys. Rev. B 344129

[21] Huldt L 1971 Phys. Status Solidi a 8173

[22] Huldt L 1974 Phys. Status Solidi a 22221

[23] Takagi S 1962 Acta Crystallogr. 151311

[24] Authier A, Malgrange C and Tournarie M 1968 Acta Crystallogr. A 24126

[25] Wie C R, Tombrello T A and Vreeland T 1991 J. Appl. Phys. 702481 